\title{
Las personas con enfermedad terminal y la necesidad de cuidados paliativos: una deuda pendiente de los servicios de salud
}

\section{Patients with terminal illness and needs for palliative care: a pending issue for healthcare services}

Correspondencia Fernando M. Runzer Colmenares frunzer@cientifica.edu.pe

Recibido: 25/03/2019

Arbitrado por pares

Aprobado: 29/05/2019

Citar como: Runzer-Colmenares FM, Parodi JF, Perez-Agüero C, Echegaray K, Samamé JC. Las personas con enfermedad terminal y la necesidad de cuidados paliativos: una deuda pendiente de los servicios de salud. Acta Med Peru. 2019;36(2):134-44

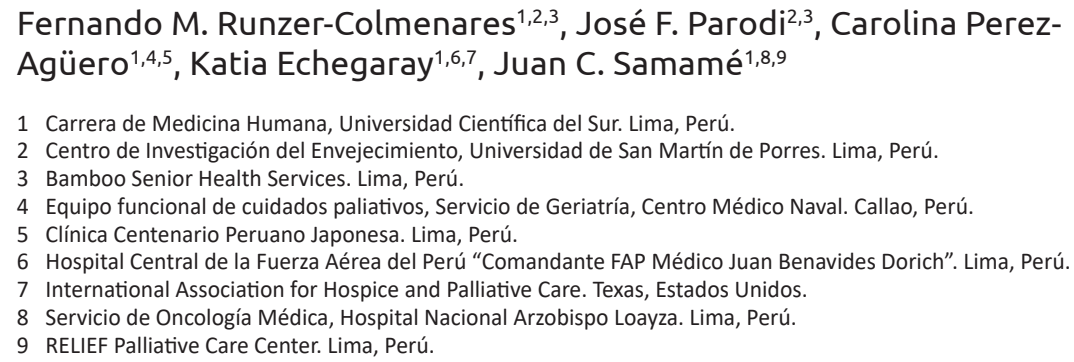

\section{RESUMEN}

Los cuidados paliativos, además de ser parte de las competencias que debería haber adquirido un profesional de la salud durante su formación, son una necesidad en crecimiento para pacientes de diferentes grupos etarios, ya sea para enfermedades oncológicas, cardiovasculares, degenerativas, entre otras. Se consideran un reto para los servicios de salud por ser cuidados complejos, que requieren de trabajo en equipo, recursos humanos y sistemas que funcionen adecuadamente. Los aspectos de gestión, salud pública, integración de servicios, dilemas éticos y aspectos legales en transición son las principales barreras a las que se enfrentan los profesionales que atienden personas con enfermedad terminal. Una de las principales armas que tenemos es consolidar programas educativos para empoderar con conocimiento a los profesionales $y$, de esta forma, que hagan notar la necesidad del manejo humano de la salud de personas con enfermedades terminales.

Palabras clave: Cuidados paliativos; Enfermo terminal; Cuidado terminal; Medicina paliativa; Programas de cuidados paliativos (fuente: DeCS BIREME). 


\section{ABSTRACT}

On top of all competences that a healthcare professional should have achieved during his/her training, palliative care is a growing need for patients from different age groups affected with cancer, cardiovascular diseases, degenerative conditions, and the like. Palliative care is a huge challenge for healthcare services, because this requires adequate teamwork, human resources and adequately functioning systems. Management, public health, service integration, ethical dilemmas, and ethical issues are the main barriers that physicians taking care of terminally ill patients must deal with. One of our main strategies for tackling this situation is to consolidate educational programs, so healthcare professionals may become empowered with knowledge; so, they may disclose the need for humanizing healthcare of terminally ill subjects.

Keywords: Palliative care; Terminally III; Terminal care; Palliative medicine; Hospice care (source: MeSH NLM).

\section{INTRODUCCIÓN}

Actualmente, las enfermedades crónicas afectan a grupos etarios cada vez más jóvenes, lo que implica que el tiempo para deteriorar la salud es mayor; además, hay un incremento en la esperanza de vida que trae como consecuencia una expasión de la morbilidad o discapacidad asociadas ${ }^{[1]}$. Esto es evidente en grupos etarios con enfermedades de pobre pronóstico, ya sean de causa cardiovascular ${ }^{[2]}$, oncológica ${ }^{[3]}$ o degenerativa ${ }^{[4]}$.

Las herramientas de los sistemas de salud han mejorado cualitativamente y la sobrevida, en líneas generales, ha aumentado ${ }^{[5]}$; sin embargo, la información sobre calidad de vida y calidad de fallecimiento de las personas con enfermedades crónicas, la infraestructura sanitaria y otros factores en el país son muy pobres ${ }^{[6]}$.

Existen aún debilidades en la atención de los centros de salud que brindan atención paliativa (AP); además, no hay un adecuado marco normativo y tanto la gestión de servicios como la capacitación de los recursos humanos son escasos. Por otro lado, la educación en salud tradicional tiende a orientar al estudiante a que la curación de la enfermedad es la única meta a alcanzar o, cuando esta no se pueda, adoptar un manejo del cuadro clínico limitado a la supervivencia del paciente.

El profesional de la salud debe hacer un tamizaje en sus pacientes y discernir quiénes son tributarios de tratamiento curativo o no, con criterios objetivos basados en evidencia, aplicados con capacitación previa y sujetos a principios bioéticos ${ }^{[7-10]}$. El código de ética del Colegio Médico del Perú relata que el profesional de medicina tiene por misión la preservación de la salud del paciente $y$, cuando no fuese posible, el alivio de las dolencias; además, en todos los casos, brindar consuelo a él y sus familiares.

La creciente cantidad de pacientes con estadios avanzados de enfermedades crónicas, enfermedades terminales (ET) y la necesidad de cuidados paliativos (CP) instan que los servicios de salud establezcan un plan de trabajo para afrontar las necesidades de atención. Esta preparación debe incluir a la educación en salud desde pregrado, donde la instrucción para brindar un manejo paliativo es prácticamente nula ${ }^{[11]}$. También capacitar al personal no sólo en temas éticos y de calidez de atención sino en el correcto diagnóstico, tamizaje, seguimiento de casos y gestión de servicios de personas con ET [12,13]. Finalmente, afianzar conocimientos bioéticos y promover la creación o adecuación de normas legales ${ }^{[14,15]}$ que faciliten la labor del personal de salud respecto al trato digno del paciente terminal y si familia.

La presente revisión tiene como objetivo dar un panorama de la situación actual sobre ET y CP, proponer un plan de trabajo multidisciplinario de forma conjunta, realzar la importancia de un manejo temprano y destacar las implicancias que podrían traer las personas con ET a nivel social, económico y sanitario, de no ejecutarse políticas y planes de salud pública sostenibles.

\section{Usuarios de servicios de salud con enfermedad terminal: requerimientos y legislación comparada}

Los requerimientos de servicios de CP varían según grupo etario y sexo; en adultos mayores y en el sexo femenino hay mayor frecuencia de necesidad de manejo paliativo ${ }^{[16]}$. Por otro lado, el cáncer y las enfermedades cardiovasculares son las que requieren $\mathrm{CP}$ de forma más frecuente ${ }^{[17]}$.

En relación con la normativa regional, en Uruguay, desde el año 2008 existen leyes que incluyen la AP. En Argentina, gobierno federado en el que cada provincia adopta de manera independiente la legislación nacional, no existe una ley de CP; en el año 2012 se aprobó la ley de "Muerte digna" que faculta a los pacientes y familiares a limitar los esfuezos terapéuticos cuando se trate de enfermedades de pobre pronóstico e irreversibles. En Brasil existe un plan nacional de CP y control de dolor crónico dictados por el Ministerio de Salud y la Agencia Nacional de Vigilancia Sanitaria y, desde 1998, son un requisito para el registro de centros de atención oncológica. En el año 2002 se incluyeron los CP en el Sistema Único de Salud y se implementaron equipos multidisciplinarios.

En Chile, la Ley de garantías explícitas de salud incorporó el Programa de alivio del dolor y CP al sistema de acceso universal. En Panamá recientemente se adoptó un programa nacional de $\mathrm{CP}$, se modificó la ley existente y se aumentó el número de días autorizados para prescribir opioides no parenterales. En Colombia se garantiza explícitamente el CP como parte del manejo integral de pacientes con cáncer y su familia; su Ley establece que los CP están orientados al mejoramiento de la 
calidad de vida de pacientes y sobrevivientes de cáncer y de sus familias. En México, en 2009 se incluyó en la Ley de Salud el derecho de recibir CP, y en 2014 se declaró la obligatoriedad de los esquemas de manejo integral de CP así como los procesos señalados en la Guía del Manejo Integral de CP ${ }^{[18]}$.

En nuestro país se creó la Ley que será base del Plan nacional de CP para enfermedades oncológicas y no oncológicas. EI Poder Ejecutivo a través del Ministerio de Salud (MINSA), en su condición de ente rector del Sistema Nacional de Salud, debe elaborar el Plan Nacional de CP para enfermedades oncológicas y no oncológicas ${ }^{[19]}$. En la elaboración del Plan Nacional, el MINSA convocó a las entidades públicas del sector, a sociedades y asociaciones científicas relacionadas a la especialidad y a las entidades privadas especializadas para que formulen aportes a su redacción. Dicho documento atiende las enfermedades crónicas progresivas que generan dependencia y constituyen amenazas para la vida, e indica que la atención ha de contener apoyo espiritual y psicológico y las medidas necesarias, con enfoque intercultural, que demandan los enfermos crónicos y terminales ${ }^{[20]}$

Adicionalemnte, en febrero de 2019 se publicó la Ley $\mathrm{N}^{\circ}$ 30681 que regula el uso medicinal y terapéutico del cannabis y sus derivados, resaltando que promueve la investigación en universidades e instituciones de investigación en salud ${ }^{[21]}$.

\section{Definiciones y conceptos básicos para la toma de decisiones}

Podemos definir el término "cuidados paliativos" como los servicios de salud destinados al alivio de problemas físicos, psicosociales y espirituales dirigidos a las personas con enfermedad terminal y las de su entorno ${ }^{[22]}$. Adicionalmnete, para comprender adecuadamante lo expuesto, la definición de ET debe ser clara. La U.S. Centers for Medicare \& Medicaid Services la define como el conjunto de procesos físicos, emocionales, sociales e intelectuales causados por una enfermedad en etapa avanzada que decrementa la "capacidad" de la persona y afecta su pronóstico y posibilidad de curación; es de naturaleza multicausal, con una expectativa de vida menor a los seis meses ${ }^{[23]}$.

Los CP tienen un abordaje interdisciplinario y se enfocan en la prevención y alivio del sufrimiento, además de brindar soporte para mantener calidad de vida en personas que tienen enfermedades de pobre pronóstico, síntomas complejos y/o ausencia de tratamiento curativo. Los servicios que brindan suelen abordar las necesidades de los usuarios (paciente y su entorno) de tal forma que los síntomas sean detectados y manejados en todas las etapas de la evolución de la enfermedad del paciente y no solo en los últimos momentos de vida del paciente como suele pensarse. Inclusive, existen modelos de atención de enfermedades potencialmente curables, en los cuales los servicios de CP tienen un rol importante para que los usuarios sobrelleven enfermedades de tratamientos largos y muchas veces agotadores de una mejor manera ${ }^{[24,25]}$, incluyendo a la familia como parte del manejo.

Por ello, es sabido que, además de un correcto manejo de molestias, síntomas físicos y psíquicos, los servicios de CP deben plantearse metas que incluyan a la evaluación y manejo integral, pactando objetivos a corto y largo plazo, orientándolas según las necesidades, creencias y evolución clínica de los usuarios. Para tal fin, es necesario contar con un equipo multidiscipliario de profesionales que cubran los aspectos psicológicos, sociales, espirituales, económicos, legales y clínicos ${ }^{[26]}$. En ese sentido, la evaluación en servicios de CP, por ser de carácter interdisciplinario, requiere del uso de escalas estandarizadas y capacitación para su correcta aplicación, además de una historia clínica detallada. Por otro lado, existe evidencia de beneficios al explorar puntos específicos en el cuidado - p.e. exploración del dolor, estado de ánimo, cognición, clínica respitratoria y gastrointestinal- con el fin de evitar quejas en la atención posteriores ${ }^{[27]}$.

En la evaluación de CP, antes que realizar una evaluación minuciosa o centrada solo en las necesidades de los usuarios, es importante determinar la fuente principal de sintomatología. Así, surgen términos como Total pain (Dolor total), con el interés de unificar el sufrimiento emocional, físico y psíquico, los temores ante la enfermedad y la ansiedad anticipatoria ${ }^{[28]}$. Si bien cuantificar objetivamente el dolor total no es factible, el hacer un abordaje que incluya todos sus dominios logra resultados positivos en relación a tolerancia al tratamiento, manejo de síntomas, entre otros ${ }^{[16,28]}$.

En este punto es preciso recordar conceptos como la eutanasia, definida como la acción de retirar la vida del ser humano por consideraciones humanísticas -evitar el sufrimiento ante una enfermedad incurable, etc-; distanasia, acción de brindar un tratamiento fútil o inútil que no brinda beneficios al paciente con ET con el fin de retrasar el proceso de morir, trayendo como consecuencia una muerte prolongada, lenta y, con frecuencia, acompañada de sufrimiento, dolor y agonía. Frente a esto surge el concepto de ortotanasia, que es el arte de morir bien, humana y correctamente, sin ser victimado por la mistanasia (muerte por abandono) o la distanasia, sin que esto implique abreviar la vida; es decir, transitar a la eutanasia ${ }^{[29]}$.

Algunas creencias religiosas también se han expresado. Juan Pablo II en su "Carta a los ancianos" escribe: "... es preciso recordar que la ley moral consiente la renuncia al llamado ensañamiento terapéutico, exigiendo sólo aquellas curas que son parte de una normal asistencia médica. Pero eso es muy diverso de la eutanasia, entendida como provocación directa de la muerte... pueden tener un papel determinante el desarrollo de la medicina paliativa, la colaboración de los voluntarios y la implicación de las familias" [30].

El judaísmo reconoce que toda la vida es finita y, como tal, sus enseñanzas son compatibles con los principios de la medicina paliativa y el cuidado al final de la vida. La tradición judía contiene dentro de sus fuentes textuales el concepto de ET. El acortamiento de la vida a través del suicidio, el suicidio asistido 
- la eutanasia está categóricamente prohibido. Aquellos pacientes con una ET pueden rechazar tratamientos que no son potencialmente curativos, especialmente cuando pueden resultar dañinos ${ }^{[31]}$.

\section{Evaluación de personas con enfermedad terminal: prevención, diagnóstico, orientación y manejo dinámicamente integral}

Los puntos débiles de los servicios de $\mathrm{CP}^{[32]}$ suelen ser diversos. La transición de un sistema sanitario a otro que incluya la atención de personas con ET y sus familias es un paso obligatorio en la actualidad, aunque puede presentar deficiencias que conlleven un mal funcionamiento ${ }^{[33]}$. Por ejemplo, en el hospital de la Marina de Guerra, Lima - Perú, la creación del Equipo funcional de CP en el Servicio de Geriatría conllevó problemas de adecuación al sistema establecido, capacitación del equipo multidisciplinario, en la gestión de citas -usualmente más de una especialidad es necesaria- y espacios físicos, la capacitación del equipo multidisciplinario y sustentación de la necesidad de la atención paliativa, entre otros. Lograr que el sistema hospitalario se adapte a un servicio de CP fue un obstáculo difícil de romper. Una estrategia para vencer este tipo de dificultades es evaluar a los casos en reuniones de equipo; así, en una sola consulta, los usuarios reciben un manejo consensuado en equipo, y durante el seguimiento, se van dosificando las atenciones ${ }^{[34]}$.

La dispensación de fármacos y la disponibilidad de estos para un tratamiento oportuno también es una barrera importante. Habitualmente, se requiere el llenado de recetarios para fármacos controlados, contar con personal auxiliar de enfermería para el recojo de los fármacos y, con previa coordinación entre farmacia y el área clínica, la disposición de fármacos urgentes para el manejo de síntomas. Por tal motivo, es necesario incluir a químicos farmacéuticos en el equipo y así tener un stock de los fármacos de rescate más usados.

La información en relación a las actitudes, conocimientos y creencias del personal de salud y los usuarios acerca de los CP y el ET es variada. Un estudio realizado a personas con cáncer concluyó que los participantes notaban una atención satisfactoria al ser atendidos en unidades de CP en comparación con el manejo habitual ${ }^{[35]}$. Otro estudio evaluó el conocimiento de estudiantes de ciencias de la salud sobre CP y concluyó que no todos los tópicos eran dominados -sobre todo respecto al manejo de síntomas ${ }^{[36]}$; además, que el nivel de empatía con el sufrimiento del paciente era alto, por ello, los autores presumen que, en futuras generaciones, la atención de estos pacientes mejorará en el tiempo ${ }^{[37]}$.

Por otro lado, existe un problema generado por la discordancia entre los síntomas reportados, la clínica descrita por el personal de salud y el tratamiento. Un estudio realizado a pacientes con diagnóstico de cáncer avanzado encontró que, si bien hubo concordancia entre lo reportado por personas y médicos, en aquellos casos en que se reportaban muchos síntomas, los médicos "decidían" cuáles eran los más importantes ${ }^{[38]}$. La sistematización de información mediante instrumentos de recolección, hitos terapéuticos y retroalimentación en equipos multidisciplinarios ayudaría a disminuir el impacto de este problema ${ }^{[39]}$. Así, en un estudio realizado con pacientes oncológicos, en el grupo de pacientes en el que el personal de salud no utilizó una entrevista estructurada había una mayor cantidad de indicaciones malinterpretadas por parte de los usuarios ${ }^{[40]}$.

Por lo tanto, cuando nos referimos a un abordaje integral, no solo hay que asegurar un manejo paliativo interdisciplinario, sino que debemos verificar el seguimiento del tratamiento con base a hitos $u$ objetivos, instrumentos y entrevistas estructuradas, sistematizando la información para propiciar la posibilidad de investigar posteriormente los hallazgos globales de las intervenciones ${ }^{[41,42]}$. En general, el contenido de la información debe contener datos sobre dolor, síntomas físicos, evaluación psicológica, antecedentes y evaluación psiquiátrica, evaluación cognitiva, comorbilidades, comprensión de la enfermedad, insight del cuadro actual, preferencias sobre el manejo y aspectos espirituales ${ }^{[43]}$.

El siguiente reto es lograr enfocar el plan en los usuarios. Para tal fin, el equipo de CP debe contar con habilidades blandas sobre comunicación, lenguaje corporal, procesos de expresión empática y entrenamiento en comunicar malas noticias ${ }^{[4,45]}$. (Figura 1).

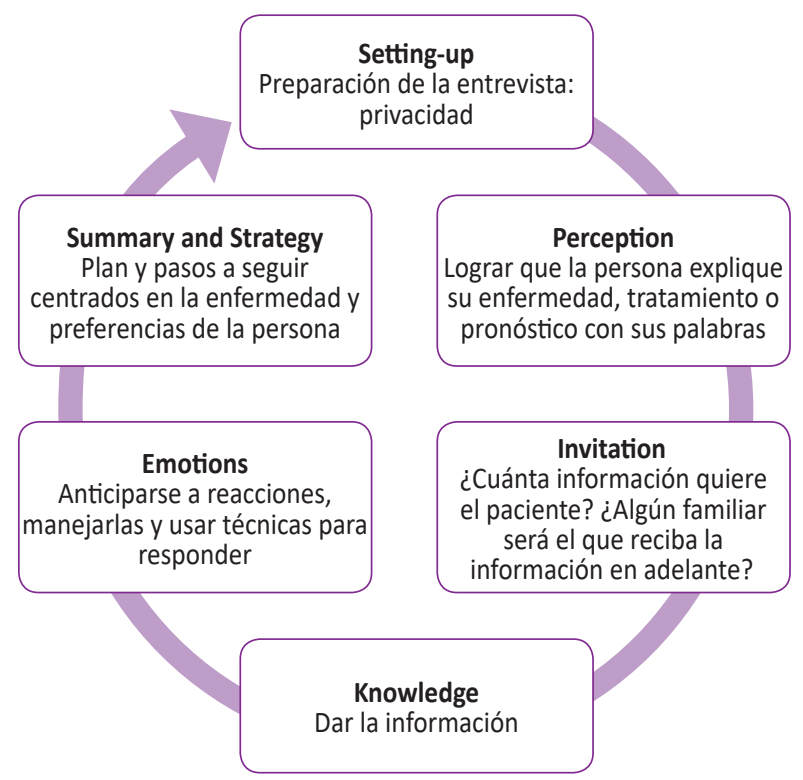

Figura 1. Protocolo SPIKES para dar malas noticias.

Buckman R. How to Break Bad News: A Guide for Health Care Professionals. Baltimore: Johns Hopkins Press; 1992.

\section{El enfoque hexagonal de cuidados de personas con enfermedad terminal: gestión integral de los problemas basados en objetivos}

Realizar la evaluación integral del paciente con ET en la primera consulta es una meta difícil de conseguir; además, conceptualmente, debería ser evaluada por diferentes miembros de un equipo. Hablamos de gestión y no sólo de evaluación 
para enfatizar que si algo se valora es para intervenir según las prioridades consensuadas entre los profesionales y las personas. Una herramienta útil es PEACE (del inglés: Physical, Emotive, Autonomy, Communication, Economic and Trascendent domains of quality of life) (ver Tabla 1) que evalúa muchas de las esferas que afectan la salud de los pacientes. Esta herramienta no tiene un punto de corte, pero sirve de "termómetro" para detectar las esferas que están alterando la calidad de vida ${ }^{[46]}$.

\section{Molestias, signos y síntomas físicos}

Las molestías físicas varían según el grupo etario, enfermedad de base y presencia de enfermedad mental. En general, las más frecuentes son depresión, ansiedad, dolor, delirium, disnea, fatiga, trastornos del sueño, náuseas/vómitos, estreñimiento, hiporexia y diarrea. Al respecto, existen herramientas para evaluar la presencia de problemas o síntomas comunes. Una de ellas es la mnemotecnia PAIN RULES (ver Tabla 2) que orienta al evaluador a revisar los síntomas más frecuentes. No sirve para clasificar riesgos ni decidir el tratamiento, pero es útil para estructurar la entrevista ${ }^{[47,48]}$. Idealmente, cada molestia identificada por el evaluador debe ser detallada en relación a antecedentes, causas, factores gatilladores, manejo y antecedente de tratamientos previos, síntomas asociados, severidad cuantitativa e importancia del síntoma para los usuarios.
Para garantizar un adecuado manejo de síntomas físicos, el personal de salud debe incluir en su plan las metas del tratamiento y las prioridades del paciente. Esto puede lograrse con escalas numéricas en relación al impacto de dichos síntomas sobre su calidad de vida, funcionalidad o estado general. Esta congruencia entre la opinión del equipo de CP y la opinión de los usuarios debe ser dinámica en el tiempo ${ }^{[49,50]}$.

\section{Comprensión de la enfermedad y preferencias: rol del "insight"}

Las personas que logran, gracias a una intervención oportuna del equipo de $C P$, una adecuada comprensión de su enfermedad una adecuada "percepción" o "visión interna" de su enfermedad - suelen brindar información más detallada sobre sus síntomas y preferencias, lo cual facilita el manejo ${ }^{[51]}$. Este punto es crucial en la evaluación integral de personas con ET, pues el equipo de CP está obligado a informar a los usuarios acerca del diagnóstico y plan, dependiendo del grado de participación que el paciente y la familia desean tener. Los principios bioéticos de autonomía y justicia deben prevalecer y se debe comunicar lo consensuado a todo el equipo. El equipo debe evaluar la capacidad de decisión de los usuarios, en especial del paciente; esto tiene incluso implicancias legales en el caso de menores de edad, personas con trastornos neurocognitivos y otros tipos de discapacidad.

Tabla 1. Herramienta PEACE ( Physical, Emotive,Autonomy, Communication, Economic and Transcendent domains of quality of life) Dominios a evaluar sobre la Calidad de vida en personas con enfermedad terminal*

\begin{tabular}{|c|c|c|c|c|c|}
\hline Físicos & Emocionales & de Autonomía & Económicos & Trascendentales & Comunicativos \\
\hline Dolor & $\begin{array}{l}\text { Debilidad, cansancio } \\
\text { o anergia }\end{array}$ & $\begin{array}{l}\text { Dependencia } \\
\text { funcional }\end{array}$ & $\begin{array}{l}\text { Sentir que requiere } \\
\text { más servicios de } \\
\text { los que el sistema } \\
\text { de salud le puede } \\
\text { brindar }\end{array}$ & $\begin{array}{l}\text { Sentirse } \\
\text { abandonado o } \\
\text { castigado por un } \\
\text { ente superior } \\
\text { (Dios) }\end{array}$ & $\begin{array}{l}\text { Sentir falta de } \\
\text { control sobre sus } \\
\text { cuidados o "no } \\
\text { entender qué es lo } \\
\text { que desea" }\end{array}$ \\
\hline $\begin{array}{l}\text { Pérdida de apetito, } \\
\text { úlceras o sequedad } \\
\text { en cavidad oral }\end{array}$ & $\begin{array}{l}\text { Insomnio o } \\
\text { hipersomnia }\end{array}$ & & $\begin{array}{l}\text { Sentir que requiere } \\
\text { más cuidados que } \\
\text { los que su familia o } \\
\text { amigos le pueden } \\
\text { brindar }\end{array}$ & $\begin{array}{l}\text { Sentir que su } \\
\text { credo, fe o culto } \\
\text { religioso no lo } \\
\text { apoya }\end{array}$ & $\begin{array}{l}\text { Sentir no estar } \\
\text { preparado o } \\
\text { temeroso por el } \\
\text { futuro }\end{array}$ \\
\hline Incontinencia & $\begin{array}{l}\text { Ansiedad, } \\
\text { nerviosismo o temor }\end{array}$ & & $\begin{array}{l}\text { Sentir que requiere } \\
\text { más servicios de } \\
\text { los que su seguro } \\
\text { de salud puede } \\
\text { coberturar }\end{array}$ & & $\begin{array}{l}\text { De la lista, ¿cuál } \\
\text { problema lo hace } \\
\text { sufrir más?** }^{*}\end{array}$ \\
\hline $\begin{array}{l}\text { Náuseas, vómitos, } \\
\text { estreñimieto } \\
\text { o síntomas } \\
\text { intestinales }\end{array}$ & $\begin{array}{l}\text { Tristeza, ánimo, } \\
\text { depresivo, } \\
\text { anhedonia, soledad, } \\
\text { desamparo }\end{array}$ & & & & $\begin{array}{l}\text { De la lista, ¿̇uál } \\
\text { problema lo hace } \\
\text { sufrir más en } \\
\text { segundo lugar?** }\end{array}$ \\
\hline Disnea o tos & $\begin{array}{l}\text { confusión, agitación } \\
\text { psicomotriz }\end{array}$ & & & & $\begin{array}{l}\text { ¿Ha tenido } \\
\text { problemas no } \\
\text { citados en la } \\
\text { lista?** }\end{array}$ \\
\hline
\end{tabular}

* Cada ítem debe ser puntuado de 0-10 ( $\mathrm{O}=$ no hay impacto en calidad de vida, 10= máximo impacto).

** Estas preguntas se hacen al final de la aplicación de la herramienta y se refieren a cualquier ítem de los dominios. 
Tabla 2. PAIN RULES: mnemotecnia para la evaluación de sintomatologia en cuidados paliativos

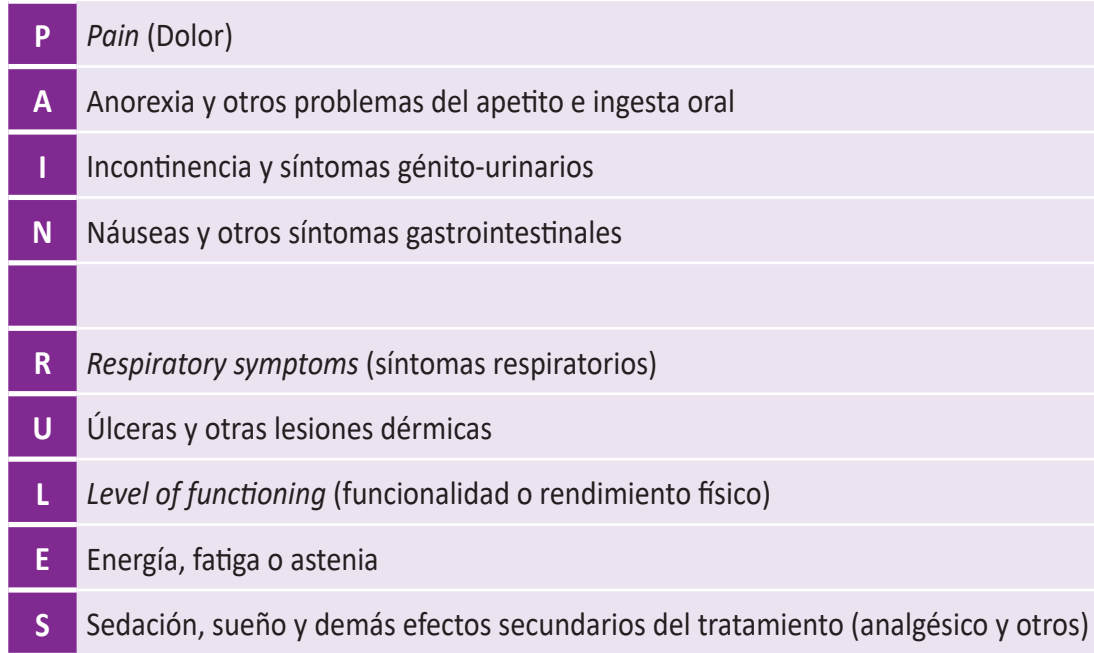

En general, debe hacerse una evaluación mental, verificar la comprensión y revisar la capacidad de escoger opciones y valorar el razonamiento y apreciación de las opciones dadas ${ }^{[52]}$.

En líneas generales, el plan se traza desde el inicio. En más de una cita, el equipo les plantea a los usuarios el plan de trabajo como parte del protocolo de información del diagnóstico. Este plan puede variar, pero los hitos o metas pactados deberían respetarse dentro de los posible, dependiendo de la evolución del paciente y otros factores ${ }^{[53]}$. Así, luego de la evaluación inicial por parte del equipo de $\mathrm{CP}$, se debe tener clara la probable trayectoria de la enfermedad, incluyendo las complicaciones más comunes, declive funcional y cognitivo y las medidas a tomarse para cada caso. Un ejemplo común es la necesidad de cesar tratamientos como quimioterapia, transfusiones, cirugías o el inicio de manejo domiciliario; hitos que el equipo tratante debería predecir, aunque no siempre es sencillo ${ }^{[54]}$.

Habitualmente, los puntos a tocar en la entrevista, cuando se va a trabajar el plan con los usuarios, incluyen la pregunta "¿qué ha entendido acerca de lo que le han dicho los médicos sobre su enfermedad/la enfermedad de su familiar?", además de anticipar respuestas emocionales, plantear situaciones futuras, alinearse con las preferencias del paciente, entre otros $^{[55]}$.

Al respecto, en muchos servicios de $\mathrm{CP}$ caen en "conspiraciones del silencio" en donde el personal sanitario, el paciente y la familia preferirían no discutir estos tópicos ${ }^{[56]}$; sin embargo, un estudio encontró que las personas con cáncer avanzado preferían una conversación abierta sobre su diagnóstico, tratamiento, opciones, riesgos y temas de decisiones compartidas ${ }^{[57]}$. Además, un estudio que incluyó conversaciones entre el equipo de CP y los usuarios mostró indicadores de una mejor calidad de vida, menor frecuencia de depresión y menor uso innecesario de unidades de terapia intensiva ${ }^{[58]}$.
Salud mental: no todo es depresión y ansiedad. Papel del "coping" en medicina paliativa

En personas con ET, la capacidad constante de adaptación a los cambios, ya sean cognitivos o de comportamiento, con miras a manejar el estrés intrínseco o extrínseco puede consumir su "reserva de recursos". Este concepto, denominado coping, es similar al de "reserva funcional" y es muy utilizado en gerontología y geriatría ${ }^{[59,60]}$. Durante la evolución del cuadro, y dependiendo de los antecedentes afectivos y cognitivos del sujeto, el paciente y la familia experimentarán problemas de depresión, ansiedad, delirium y declive de funciones cognitivas, ya sea por toxicidad farmacológica, evolución de la enfermedad de fondo, pobre reserva funcional neurológica o una combinación de las anteriores ${ }^{[61]}$.

Los clínicos responsables deben hacer una evaluación exhaustiva sobre medicación, trastornos electrolíticos, metabólicos o nutricionales, cognición, problemas económicos-sociales, dolor, entre otros y manejarlos, pues los trastornos del ánimo pueden ser secundarios.

En lo que respecta a la depresión, suele ser el más frecuente en ET, caracterizada por "dolor emocional", desmoralización, entre otros componentes clásicos de trastornos depresivos. Es importante diferenciar el duelo anticipado, el sufrimiento comprensible por el diagnóstico y la depresión. Se debe evitar medicar a personas que no tengan un diagnóstico certero ni un manejo de equipo. Sin embargo, existe evidencia que la depresión está presente en casi el $40 \%$ de personas con enfermedades terminales ${ }^{[62]} y$ su impacto logra incluso a acelerar el fallecimiento, entre otros desenlaces negativos ${ }^{[63]}$.

Sin importar cuál instrumento se utilice, se recomienda realizar tamizajes periódicos de depresión en personas con ET. Preguntas cortas como "¿se ha sentido triste o deprimido en las últimas dos semanas?" son bastante útiles en contextos de enfermedad 
avanzada. Luego del tamizaje, se deben seguir criterios gold standard de diagnóstico y tratamiento, tomando en consideración las interacciones farmacológicas, vías de administración de fármacos y viabilidad de adherencia ${ }^{[64]}$. Adicionalmente, se recomienda evaluar el impacto de la depresión en la vida diaria, calidad de vida relacionada a salud mental y sintomatología específica como desmoralización, sufrimiento, trastornos del sueño, entre otros ${ }^{[65]}$.

En relación con trastornos de ansiedad, la evaluación debe incluir historia mental previa, abuso de sustancias, uso actual de psicofármacos, delirium, dolor, y trastornos metabólicos, además del entorno sociofamiliar. El tamizaje con instrumentos o preguntas puntuales es recomendado, como, por ejemplo: "¿¿en las últimas dos semanas ha tenido molestias como nerviosismo, ansiedad, angustia o incapacidad de poder dejar de preocuparse por algo?" [66]. El tratamiento debe ser, al igual que en todos los casos de enfermedades mentales, evaluados en equipo y escoger la medicación con menos efectos adversos.

Para el caso de delirium, el personal se enfrenta a un cuadro frecuente y potencialmente reversible en más de la mitad de las ocasiones, pero con una alta tasa de recurrencia. Por definición, es secundario a una causa de base, ya sea infecciosa, metabólica, hidroelectrolítica o por fármacos, lo que implica que su existencia indica que la reserva funcional cognitiva ya está venida a menos. Se caracteriza por ser de inicio agudo, curso fluctuante, con déficit de atención y nivel de conciencia, además de pensamiento desorganizado ${ }^{[67]}$.

Como parte de la aplicación de lo principios éticos sobre autonomía y beneficencia, surge la necesidad de valorar la capacidad mental de decidir de la persona con enfermedad terminal. Sin embargo, no se dispone de ninguna herramienta de aplicación universal que pueda evaluar de manera válida la capacidad de toma de decisiones de las personas, sobretodo si ya tienen algún grado de deterioro cognitivo en las que se requiere una evaluación más detallada de la capacidad para tomar decisiones. Por lo tanto, es necesaria la validación de herramientas para documentar la capacidad de tomar decisiones en estas condiciones ${ }^{[68]}$.

\section{Evaluaciones de soporte: religiosidad y espiritualidad}

Un problema de salud afecta en muchos ámbitos al ser humano y su trascendencia puede calar en los sentimientos, traspasando barreras físicas y afectivas. La evaluación de este punto es importante y compleja. Sin una adecuada preparación, el personal de salud va a ser tomado por sorpresa con preguntas como “¿por qué a mí?" o “¿morir duele?". Independientemente de si los usuarios son agnósticos, ateos o creyentes, en situaciones cercanas a la muerte, aparecen cuestiones existenciales que el equipo de CP debe estar preparado para manejar. Tal vez no podrán responder, pero si saber conducir la entrevista ${ }^{[69]}$.

Para estos casos, la calidez, la empatía, la experiencia y los conocimientos son necesarios, pero no suficientes. Existen publicaciones que demuestran que los familiares y personas con cáncer muy avanzado que tienen creencias religiosas muy arraigadas suelen requerir con mayor frecuencia de unidades de cuidados intensivos cuando el momento del fallecimiento es cercano. Además, otro estudio habla que los oncólogos suelen ser los médicos que menos abordan este punto, lo que se asociaría con mayores intervencionismos fútiles en personas con ET ${ }^{[70,71]}$. En países como el nuestro, donde los usuarios van a recibir información de sus amistades, familiares, medios de comunicación y redes sociales acerca de tratamientos "milagrosos" - como el bicarbonato de sodio, vitaminas, medicina natural, biomagnetismo, homeopatía, entre otros -, el personal de CP debe tener respuestas enfáticas sin despreciar la opinión del paciente [72]. Se debe preguntar por la importancia de la espiritualidad y religiosidad en el paciente y su entorno. Síntomas afectivos y físicos podrían verse aliviados por la presencia de representantes de credos. En países donde se practica la eutanasia, las crisis de fe son frecuentes, pero no son una cuestión exclusiva de esos países ${ }^{[73]}$.

\section{Ámbito socioeconómico}

Así el paciente tenga un seguro con gran cobertura, la enfermedad avanzada genera gastos habitualmente no medidos y muchas veces afecta a integrantes de la familia que solventaban parte de los gastos del hogar. Por otro lado, sabemos que no siempre las personas tienen cobertura adecuada, por lo que la repercusión económica y social es grande.

El soporte necesario para un paciente con ET requiere de tiempo de los familiares, ya sea de cuidado en casa o en centros de salud, gastos de cuidadores, medicamentos, traslados, entre otros. Tanto el paciente como los familiares deben reducir sus horas laborales por causa de la enfermedad, lo que ocasiona serios déficits económicos. Por ello, el área de Asistencia social debe formar parte del equipo de $\mathrm{CP}$, para evaluar el núcleo familiar, recursos económicos, tipo de cobertura de salud, etc. Esta evaluación es sumamente importante pues, de ser ignorada, impacta negativamente en la salud, calidad de vida y adecuación de plan de cuidados ${ }^{[74,75]}$.

\section{Continuidad de cuidados: niveles asistenciales}

El alta de un servicio y la transferencia a otro han sido un dolor de cabeza para gestores de salud por años. La complejidad de los requerimientos asistenciales de las personas con ET es altísima. Así, poniendo como ejemplo a nuestro fraccionado sistema de salud y la gran cantidad de trámites administrativos que deben realizar los usuarios y el personal, garantizar la coordinación adecuada para el cuidado entre servicios y niveles asistenciales es una tarea titánica, pero no imposible ${ }^{[76,77]}$. Existen numerosas publicaciones en las que se describen las características de los modelos de cuidados transicionales en CP en comparación con los cuidados habituales, así como las barreras y facilitadores para su implementación ${ }^{[78]}$.

En los periodos de referencia y contrarreferencia la probabilidad de iatrogenia es mayor, en especial en personas con problemas cognitivos u otras discapacidades, asociándose a uso de recursos hospitalarios distintos a los CP y que no se relacionaban con el 
plan trazado. La evaluación integral en CP obliga a individualizar la gestión hospitalaria para personas con ET, no sólo por el bien del usuario, sino que también para hacer más eficiente la gestión de los servicios. Para ello, se recomienda dar por escrito indicaciones de transferencia o referencia, dar indicaciones claras con relación a requerimiento de servicios de CP de forma aguda, brindar al usuario la oportunidad de comunicación telefónica y, lo más importante, que todo el equipo conozca la logística del centro de salud ${ }^{[79,80]}$. Los programas no sólo pueden guiar la implementación sino también la evaluación y procesos de mejora continua de éstos ${ }^{[81\}}$.

\section{Programas educativos sobre cuidados paliativos y enfermedad terminal}

La formación académica es importante en el desarrollo de los CP para impulsar un servicio de calidad. Para dar respuesta a esta necesidad, puesto que la complejidad, el ámbito de aplicación y la cobertura asistencial van a ser diferentes, se requieren distintos niveles de formación. De esta manera, se ha ideado un plan de formación basado en cuatro escalones o niveles que se han de ir adquiriendo y superando hasta alcanzar el grado de experto ${ }^{[82]}$.

Por ello, los programas educativos en pregrado, elaborados por universidades e instituciones de educación superior en el área de la salud y disciplinas afines, deberían incorporar la asignatura y/o contenidos de cuidados paliativos en el currículo, según el plan de estudios ${ }^{[83\}}$. Actualmente nuestro país se encuentra en un proceso de reestructuración curricular y aun son muy pocas las universidades que han incluido esta materia dentro de su plan curricular, aún considerada como un capitulo dentro de una asignatura, y no una asignatura per se. Según la escala de niveles formativos, actualmente se cuentan con planes de formación presencial en la modalidad de diplomados. Al respecto, cabe mencionar que contamos con asociaciones y sociedades afines a CP en Perú: Sociedad Peruana de Cuidados Paliativos, Asociación Médica de Cuidados Paliativos y La Red Internacional de Enfermeras en Cuidados Paliativos, que contribuyen con la formación continua de los profesionales.

\section{Hoja de ruta para el desarrollo de la atención paliativa: los pasos siguientes}

Los avances en la ciencia médica y en el marco legal relacionados a la atención paliativa deben encontrar una respuesta en un modelo de atención de salud nacional que integre la atención paliativa en todos los niveles de atención, bajo la observancia del principio de cobertura universal de salud.

El desarrollo de un Plan Nacional de CP debe tener como eje el cuidado de la persona y la familia, con enfoque de curso de vida y en el marco de un sistema de salud liderado por la atención primaria. Un sistema de asistencia paliativa sostenible, de calidad adecuada y accesible debe integrarse en el contexto de la atención primaria de la salud líder, la atención comunitaria y domiciliaria, y los proveedores de cuidados de apoyo, como familiares y voluntarios de la comunidad.
La atención paliativa tiene su mayor beneficio cuando las intervenciones son tempranas, de ahí la necesidad de su desarrollo en el primer nivel de atención. Esto significa que la atención paliativa no debe circunscribirse a especialistas ni centros de alta complejidad ${ }^{[84]}$.

Consideramos que la gestión del comportamiento humano, a través de programas de capacitación, es clave para el cambio organizacional. La capacitación del recurso humano no sólo se refiere a los profesionales de salud, sino también a los gestores, promotores, cuidadores y a los usuarios. Así mismo y de la mano de la capacitación acreditada y la readecuación de los servicios sobre todo del primer nivel de atención, se requiere la creación de las condiciones que garanticen el acceso seguro a medicamentos que permitan controlar eficazmente síntomas como el dolor ${ }^{[85]}$.

Por último, la necesidad de intervenciones basadas en evidencia nos exige a crear evidencia propia sobre la cual sustentar y optimizar nuestras intervenciones. Para esto es fundamental incluir en el sistema de información de salud indicadores de proceso e impacto relacionados con atención paliativa. En este sentido, la promoción y desarrollo de investigaciones relacionadas con el tema por instituciones académicas y de salud pública es un imperativo ${ }^{[86,87]}$.

Actualmente la atención paliativa en nuestro país vive un proceso de mejora a partir de diversas oportunidades que se dan hoy en día como normativas, educación y sensibilización por parte de nuestras autoridades estatales y población.

Contribución de los autores: Todos los autores participaron en la concepción, redacción, búsqueda bibliográfica y aprobación de la versión final del manuscrito.

\section{Financiamiento: Autofinanciado}

Conflictos de interés: todos los autores declaran no tener conflictos de interés.

\section{REFERENCIAS BIBLIOGRÁFICAS}

1. Robine J. Looking forward to a general theory on population aging. J Gerontol A Biol Sci Med Sci. 2004;59(6):M590-7.

2. Markgren R, Brännström $\mathrm{M}$, Lundgren $\mathrm{C}$, Boman K. Impacts of person-centred integrated chronic heart failure and palliative home care on pharmacological heart failure treatment: a substudy of a randomised trial. BMJ Support Palliat Care. 2019;9(1):e10.

3. Runzer-colmenares F, Runzer-colmenares FM, Urrunaga-pastor $D$, Aguirre LG. Frailty and vulnerability as predictors of radiotoxicity in older adults: A longitudinal study in Peru Frailty and vulnerability as predictors of radiotoxicity in older adults: A longitudinal study in Peru. Med Clin (Barc). 2017;149(8):325-30.

4. Parodi JF, Nieto-Gutierrez W, Tellez WA, Ventocilla-Gonzales I, Runzer-Colmenares, Fernando M. Taype-Rondan A. Gait speed and the appearance of neurocognitive disorders in older adults: Results of a Peruvian cohort. Rev Esp Geriatr Gerontol. 2018;53(2):8-11. 
5. Higginson IJ. Research challenges in palliative and end of life care. BMJ Support Palliat Care. 2016 Mar;6(1):2-4.

6. Chen CY, Naessens JM, Takahashi PY, Mccoy G, Borah BJ, Borkenhagen LS, et al. Improving value of care for older adults with advanced medical illnessand functional decline: cost analyses of a home-based palliative careprogram. J Pain Symptom Manage. 2018;56(6):928-35.

7. Fortín-Magana MA, Portillo-Santamaría K, Gómez-Casanovas J, López-Saca M. Enseñanza de los cuidados paliativos en las escuelas de medicina de El Salvador. Educación Médica. 2019;Supl 1:143-7.

8. Marques de Sanvezzo V, Santiago-Montandon D, Ferreira-Esteves LS. Instruments for the functional assessment of elderly persons in palliative care: an integrative review. Rev Bras Geriatr Gerontol. 2018;21(5):604-15.

9. Gomez-Batiste X, Blay C, Antoni-Broggi M, Lasmarias C. Ethical challenges of early identification of advanced chronic patients in need of palliative care: the Catalan experience. J Palliat Care. 2018;33(4):247-51.

10. Sandman L, Molander U, Benkel I. Developing organisational ethics in palliative care: A three-level approach. Nurs Ethics. 2015;24(2):138-50.

11. Wilson J, Hodgson J, Jones E, Wilson G. Medical family therapy in spiritual care. In: Mendenhall T, Lamson A, Hodgson J, Baird M (eds). Clinical methods in medical family therapy. Springer, Cham; 2018. p. 463-95.

12. Moody K, McHugh M, Baker R, Cohen H. Providing pediatric palliative care education using problem-based learning. J Palliat Med. 2017;21(1):22-7.

13. Malloy P, Takenouchi S, Kim HS, Lu Y, Ferrell B. Providing palliative care education: showcasing efforts of Asian nurses education is key: a palliative care. Asia Pac J Oncol Nurs. 2018;5:15-20.

14. Ezer T, Burke-shyne N, Hepford K. Legal support for palliative care patients. J Pain Symptom Manage. 2018;55(2):S157-62.

15. Dias-Reis-Pessalacia J, Pinto S, Resende-Boaventura J, MenisFerreira A. Bioethics and palliative care in primary health care [Internet]. In: Morales-González JA, Aguilar Nájera ME (ed). Reflections on Bioethics. London, IntechOpen; 2018 [citado el 14 de enero de 2019]. p. 75-84. Disponible en: https://www.intechopen. com/page/contact-us

16. Connor SR, Sepulveda Bermedo MC (ed). Global atlas of palliative care at the end of life. Connor SR, editor. London: Worldwide Palliative Care Alliance; 2014.

17. Kierner KA, Weixler D, Masel EK, Gartner V, Watzke HH. Polypharmacy in the terminal stage of cancer. Support Care Cancer. 2016;24(5):2067-74.

18. Pastrana T, De Lima L, Wenk R, Eisenchlas J, Monti C, Rocafort J, et al. Atlas de Cuidados Paliativos en Latinoamérica. Houston: International Association for Hospice and Palliative Care; 2012.

19. Congreso de la República. Ley $N^{\circ} 30846$ : Ley que crea el plan nacional de cuidados paliativos para enfermedades oncológicas y no oncológicas. Lima, Perú; 2018.

20. Ministerio de Salud de Perú. Plan Esperanza: Plan Nacional para la Atención Integral del Cáncer. Lima: MINSA; 2015.

21. Congreso de la República. Ley $N^{\circ} 30681$ : Ley que regula el uso medicinal y terapéutico del Cannabis y sus derivados. Lima, Perú; 2017.

22. Krakauer EL. Palliative care, toward a more responsive definition. Textb Palliat Care. 2018:1-5.
23. Office of External Affairs. Centers for Medicare and Medicaid Services. United States of America: U.S. Department of Health and Human Services; 2018.

24. Ottolini L, Keller N, Fusi-schmidhauser T, Oriani A, Gamondi C. Innovative models of palliative care delivery: integrated palliative care in Internal Medicine Hospital. J Pain Symptom Manage. 2018;56(6):e108.

25. Driessen J, West T. Recent Evidence on the Inclusion of Hospice and Palliative Care Physicians in Medicare Shared Savings Program. J Palliat Med. 2017:1-3.

26. Loh KP, Abdallah M, Shieh M, Stefan MS, Pekow PS, Lindenauer $\mathrm{PK}$, et al. Use of inpatient palliative care services in patients with advanced cancer receiving critical care therapies. J Natl Compr Canc Netw. 2018;16(9):1055-64.

27. Dhingra L, Barrett $M$, Knotkova $H$, Chen J, Riggs A, Lee B, et al. Symptom distress among diverse patients referred for communitybased palliative care: sociodemographic and medical correlates. J Pain Symptom Manage. 2018;55(2):290-6.

28. Mehta A, Chan LS, Saunders C. Understanding of the Concept of Total Pain A Prerequisite for Pain Control. J Hosp Palliat Nurs. 2008;10(1):26-32.

29. Amorim-Biondo C, Paes da Silva MJ, Dal Secco LM. Distanásia, eutanásia e ortotanásia: percepções dos enfermeiros de unidades de terapia intensiva e implicações na assistência. Rev Latino-Am Enfermagem. 2009;17(5):613-9.

30. Vaticano. carta del Santo Padre Juan Pablo II a los ancianos. Vaticano; 1999.

31. Kinzbrunner BM. Review Article Jewish Medical Ethics and End-ofLife Care. J Palliat Med. 2004;7(4):558-73.

32. Johnson DMW. Acceptability of the MD Anderson Symptom Inventory and qualitative analysis of barriers to palliative care services for rural and non-rural populations. Kirkhof College of Nursing; 2017.

33. Ronaldson S, Devery K. The experience of transition to palliative care services: Perspectives of patients and nurses. Int J Palliat Nurs. 2001;7(4):171-7.

34. Sorensen AM, Wentlandt K, Krzyzanowska MK, Le LW, Rodin G, Zimmermann C. Early palliative care: Attitudes and opinions of palliative care physicians. J Clin Oncol. 2017;34(15):e21669

35. Rioja-García BS, Cervera-Vallejos MF. Percepción sobre el tratamiento paliativo en personas con enfermedad oncológica avanzada. Acc Cietna. 2018;5(2):57-65.

36. Ordoñez Molero DA, Rivera-Muñoz AE, Mateluna Paredes PC, Málaga-Rodriguez G. Nivel de conocimientos acerca de cuidados paliativos en alumnos de medicina de sexto año de la Universidad Peruana Cayetano Heredia, Lima, Perú, año 2018 [Tesis Bachiller]. Liam, Perú: Universidad Peruana Cayetano Heredia; 2018.

37. Solano-Solano AA, Bazán-Sánchez AC. Experiencias de las estudiantes de enfermería en el cuidado a la persona al final de la vida - Chiclayo, Perú. 2016. Acc Cietna. 2018;5(1):51-62.

38. Strömgren AS, Groenvold M, Pedersen L, Olsen AK, Spile M, Sjogren $P$. Does the medical record cover the symptoms experienced by cancer patients receiving palliative care? A comparison of the record and patient self-rating. J Pain Symptom Manage. 2001;21(3):189-96.

39. Mehta DH, Perez GK, Traeger L, Park ER, Goldman RE, Haime V, et al. Building resiliency in a palliative care team: a pilot study. J Pain Symptom Manage. 2016;51(3):604-8.

40. Mack JW, Weeks JC, Wright AA, Block SD, Prigerson HG. Endof-life discussions, goal attainment, and distress at the end of 
life: predictors and outcomes of receipt of care consistent with preferences. J Clin Oncol. 2010;28(7):1203-8.

41. Scandrett KG, Reitschuler-Cross EB, Nelson L, Sanger JA, Feigon $M$, Boyd $E$, et al. Feasibility and effectiveness of the NEST13+ as a screening tool for advanced illness care needs. J Palliat Med. 2010;13(2):161-9.

42. Henoch I, Carlander I, Holm M, L, James I, Lecturer RNS, et al. Palliative Care Research - A Systematic Review of foci, designs and methods of research conducted in Sweden between 2007 and 2012. Scand J Caring Sci. 2016;30(1):5-25.

43. Best M, Butow P, Olver I. Palliative care specialists' beliefs about spiritual care. Support Care Cancer. 2016;24(8):3295-306.

44. Forbat L, Hons BA, Cert PG, Franc K, Callaghan LO, Kulikowski J. Family meetings in inpatient specialist palliative care: a mechanism to convey empathy. J Pain Symptom Manage. 2018;55(5):1253-9.

45. Carver $C$. The fear of dying: a case study using the SPIKES protocol. Fam Soc J Contemp Soc Serv. 2018;99:333-7.

46. Bellamy G, Stock J, Schofield P. Health care professionals' views associated with the barriers and facilitators of advance care planning (ACP) for community dwelling older adults with palliative and end-of-life care needs towards achieving a 'good' death: Findings from a qualitative, exploratory pilot study. Geriatrics. 2018;3:1-12.

47. Solano JP, Gomes B, Higginson IJ, Bs BM. A Comparison of symptom prevalence in far advanced cancer, aids, heart disease, chronic obstructive pulmonary disease and renal disease. 2006;31(1):58-69.

48. Moens K, Higginson IJ, Harding R. Are there differences in the prevalence of palliative care-related problems in people living with advanced cancer and eight non-cancer conditions? A systematic review. J Pain Symptom Manag. 2014;48(4):660-77.

49. Friedel $\mathrm{M}$, Aujoulat I, Dubois A-C, Degryse J-M. Instruments to measure outcomes in pediatric palliative care: a systematic review. Pediatrics. 2019;143(1):e20182379.

50. Mercadante S, Adile C, Lanzetta G, Mystakidou K, Maltoni M, Guilherme-Soares $L$, et al. Personalized symptom goals and patient global impression on clinical changes in advanced cancer patients. Oncologist. 2019;24(2):239-46.

51. Bulk LY, Kimel G, Bates J, King N, Nimmon L. Relational dimensions of quality of life in inpatient palliative facilities. J Pain Symptom Manage. 2018;56(6):e35-6.

52. Santoro JD, Bennett M. Ethics of end of life decisions in pediatrics: a narrative review of the roles of caregivers, shared decision-making, and patient centered values. 2018;8(5).

53. Akard TF, Hendricks-ferguson VL, Gilmer MJ. Pediatric palliative care nursing. Ann Palliat Med. 2019;8(Suppl 1):S39-48.

54. Forbat L, Chapman M, Lovell C, Liu W, Johnston N. Improving specialist palliative care in residential care for older people: a checklist to guide practice. BMJ Support Palliat Care. 2018;8(3):34753.

55. Halporn JD, Rinaldi SP, Jackson VA. Ambulatory Palliative Care. Qual Cancer Care. 2018;217-42.

56. Tortolero NM. Conspiración de silencio: ¿ayuda o agonía? = Conspiracy of silence: aid or agony? 2018;9(2):230-6.

57. Enzinger AC, Zhang B, Schrag D, Prigerson HG, Enzinger AC. Outcomes of prognostic disclosure: associations with prognostic understanding, distress, and relationship with physician among patients with advanced cancer. J Clin Oncol. 2015;33(32):3809-16.

58. Wright AA, Zhang B, Ray A, Mack JW, Trice E, Balboni T, et al. Associations between end-of-life discussions, patient mental health, medical care near death, and caregiver bereavement adjustment. JAMA. 2010;300(14):1665-73

59. Birgitte A, Ammari H, Hendriksen C, Rydahl-hansen S. Results from the family and coping oriented palliative homecare intervention study (FamCope)- A randomized controlled trial. J Psychosoc Oncol. 2018;36(5):557-81.

60. Atamna H, Tenore A, Lui F, Dhahbi JM. Organ reserve, excess metabolic capacity, and aging. Biogerontology. 2018;19(2):171-84

61. Brunello A, Lombardi G, Zagonel V. Rehabilitation treatment in older cancer patients. En: Masiero E, Carraro U. Rehabilitation Medicine in Elderly Patients. Italia: Springer Intl Publishing; 2018. p. 503-510.

62. Mitchell AJ, Chan M, Bhatti H, Halton M, Grassi L, Johansen C, et al. Prevalence of depression, anxiety, and adjustment disorder in oncological, haematological, and palliative-care settings: a meta-analysis of 94 interview-based studies. Lancet Oncol. 2011;12(2):160-74.

63. Satin JR, Linden W, Phillips MJ. Depression as a Predictor of Disease Progression and Mortality in Cancer Patients. Cancer. 2009;115(22):5349-61.

64. Brenne E. Depression in palliative care cancer patients [Thesis Doctor]. Norwegian University of Science and Technology Faculty; 2018.

65. Akizuki N, Yamawaki S, Akechi T. Development of an impact thermometer for use in combination with the distress thermometer as a brief screening tool for adjustment disorders and/or major depression in cancer patients. J Pain Symptom Manag. 2005;29(1):91-9.

66. Götze H, Brähler SE, Gansera L, Schnabel A, Gottschalk-Fleischer A Köhler N. Anxiety, depression and quality of life in family caregivers of palliative cancer patients during home care and after the patient' s death. Eur J Cancer Care (Engl). 2018;27(2):e12606.

67. Centeno C, Sanz A, Bruera E. Delirium in advanced cancer patients Palliat Med. 2004;18(3):184-94.

68. Pennington C, Davey K, Meulen R-T, Coulthard E, Kehoe P-G. Tools for testing decision-making capacity in dementia. Age Ageing. 2018;47(6):778-84.

69. Balducci L. Geriatric oncology, spirituality, and palliative care. J Pain Symptom Manage. 2019;57(1):171-5.

70. Phelps AC, Maciejewski PK, Nilsson M, Tracy A, Wright AA, Paulk $M E$, et al. Association between religious coping and use of intensive life- prolonging care near death among patients with advanced cancer. JAMA. 2009;301(11):1140-7.

71. Rodin D, Balboni M, Mitchell C, Smith PT, Vanderweele TJ, Balboni TA. Whose role? Oncology practitioners' perceptions of their role in providing spiritual care to advanced cancer patients. Support Care Cancer. 2015;23(9):2543-50.

72. Zevallos-Morales A, Quiñonez-Laveriano DM, Taype-Rondan A. Cursos de terapias pseudocientíficas auspiciados por el Colegio Médico del Perú. Acta Med Peru. 2016;15(2):155-6.

73. Dierickx S, Deliens L, Cohen J, Chambaere K. Involvement of palliative care in euthanasia practice in a context of legalized euthanasia: A population-based mortality follow-back study. Palliat Med. 2018;32(1):114-22.

74. Glajchen M, Berkman C, Otis-green S, Stein GL, Sedgwick T, Bern-klug $\mathrm{M}$, et al. Defining core competencies for generalistlevel palliative social work. J Pain Symptom Manage. 2018;56(6):886-92.

75. Reese DJ, Csikai EL. Social work assessment and outcomes measurement in hospice and palliative care. Am J Hosp Palliat Med. 2018;35(12):1553-64. 
76. Slater PJ, Edwards RM. Needs analysis and development of a staff well-being program in a pediatric oncology, hematology, and palliative care services group. J Healthc Leadersh. 2018;10:55-65.

77. Mccorry NK, Connor SO, Leemans K, Coast J, Donnelly M, Finucane $A$, et al. Quality indicators for palliative day services: a modified Delphi study. Palliat Med. 2019;33(2):197-205.

78. Ng AY, Wong FK, Lee PH. Effects of a transitional palliative care model on patients with end-stage heart failure: study protocol for a randomized controlled trial. Trials. 2016;17:173.

79. Vallianatos S, Huizinga C, Gijzen R, Schuiling-otten M, Verhagen E. Nationwide structure for integrated children palliative care. Int J Integr Care. 2018;18:90.

80. Mertens F, Pype P, Deveugele M. Healthcare professionals' experiences of interprofessional collaboration during palliative patients' transfer of care setting: a focus group study. Int J Integr Care. 2018;18:262.

81. Smith S, Brick A, Hara SO, Normand C. Evidence on the cost and cost-effectiveness of palliative care: A literature review. Palliat Med. 2014;28(2):130-50
82. Teresa M, Merino G, Gil E, Consuelo M, Gómez F, María E, et al. Educación Médica Programa centralizado de formación en Cuidados. Educación Médica. 2017;18(2):103-13.

83. Parodi JF, Morante R, Hidalgo L, Carreño R. Propuesta de políticas sobre cuidados paliativos para personas adultas mayores en Latinoamérica y el Caribe. Horiz Med. 2016;16(1):69-74.

84. Ofran Y, Bar-sela G, Toledano M, Kushnir I, Moalem B, Gil W, et al. Palliative care service incorporated in a hematology department: a working model fostering changes in clinical practice. Leuk Lymphoma. 2019;60(8):2079-81.

85. Mizuno A, Shibata T, Oishi S. The Essence of Palliative Care Is Best Viewed as the "Problematization". J Palliat Med. 2019;22(1):6.

86. García JS, Brull CO, Méndez JJDA, Pascual MC. Palliative medicine for patients with advanced heart failure: New evidence. Rev Clin Esp. 2019. Article in press Available from: https://doi.org/10.1016/j. rceng.2018.09.010

87. Chung A, Collier A, Gott M. Community-led and/or focused initiatives to support family carers within a palliative care context: An integrative review. Palliat Med. 2019;33(2):250-6.

\title{
Las ediciones anteriores de Acta Médica Peruana están disponibles en:
}

\author{
www.redalyc.org
}

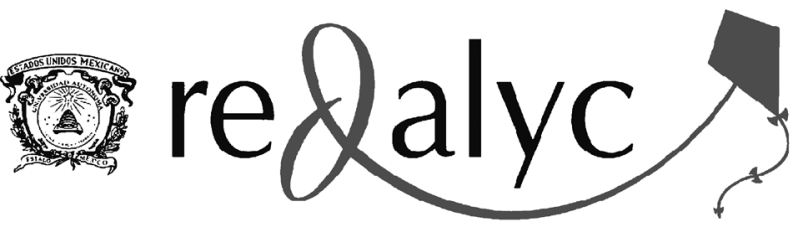

